\title{
Inflow of atomic gas fuelling star formation
}

M. J. Michałowski ${ }^{1}$, G. Gentile ${ }^{2,3}$, J. Hjorth ${ }^{4}$, M. R. Krumholz ${ }^{5}$, N. R. Tanvir ${ }^{6}$, P. Kamphuis ${ }^{7}$, D. Burlon ${ }^{8}$, M. Baes ${ }^{2}$, S. Basa ${ }^{9}$, S. Berta ${ }^{10}$, J. M. Castro Cerón ${ }^{11}$, D. Crosby ${ }^{1}$, V. D'Elia ${ }^{12,13}$, J. Elliott ${ }^{10}$, J. Greiner ${ }^{10}$, L. K. Hunt ${ }^{14}$, S. Klose ${ }^{15}$, M. P. Koprowski ${ }^{1}$, E. Le Floc'h ${ }^{16}$, D. Malesani ${ }^{4}$, T. Murphy ${ }^{8}$, A. Nicuesa Guelbenzu ${ }^{15}$, E. Palazzi ${ }^{17}$, J. Rasmussen ${ }^{4,18}$, A. Rossi ${ }^{17,15}$, S. Savaglio ${ }^{19,20}$, P. Schady $^{10}$, A. de Ugarte Postigo ${ }^{21,4}$, D. Watson ${ }^{4}$, P. van der Werf $^{22}$, S. D. Vergani ${ }^{23,24}$ and D. $\mathrm{Xu}^{4}$

${ }^{1}$ Institute for Astronomy, University of Edinburgh, Royal Observatory, Edinburgh, UK ${ }^{2}$ Sterrenkundig Observatorium, Universiteit Gent, Krijgslaan 281-S9, 9000, Gent, Belgium

${ }^{3}$ Department of Physics and Astrophysics, Vrije Universiteit Brussel, Pleinlaan 2, Belgium

${ }^{4}$ Dark Cosmology Centre, Niels Bohr Institute, University of Copenhagen, Denmark

${ }^{5}$ Department of Astronomy and Astrophysics, University of California, Santa Cruz, USA

${ }^{6}$ Department of Physics and Astronomy, University of Leicester, UK

${ }^{7}$ CSIRO Astronomy \& Space Science, Australia Telescope National Facility, Epping, Australia

${ }^{8}$ Sydney Institute for Astronomy, School of Physics, The University of Sydney, Australia

${ }^{9}$ Aix Marseille Université, CNRS, LAM (Laboratoire d'Astrophysique de Marseille), France

${ }^{10}$ Max-Planck-Institut für Extraterrestrische Physik, Giessenbachstraße, Garching, Germany

${ }^{11}$ ISDEFE for the Herschel Science Centre (ESA-ESAC), Villanueva de la Cañada, Spain

${ }^{12}$ ASI-Science Data Center, Via del Politecnico snc, Rome, Italy

${ }^{13}$ INAF - Osservatorio Astronomico di Roma, Via di Frascati, Monteporzio Catone, Italy

${ }^{14}$ INAF-Osservatorio Astrofisico di Arcetri, Largo E. Fermi 5, Firenze, Italy

${ }^{15}$ Thüringer Landessternwarte Tautenburg, Sternwarte 5, Tautenburg, Germany

${ }^{16}$ Laboratoire AIM-Paris-Saclay, CEA, France

${ }^{17}$ INAF-IASF Bologna, Via Gobetti 101, Bologna, Italy

${ }^{18}$ Technical University of Denmark, Department of Physics, Kgs. Lyngby, Denmark

${ }^{19}$ Physics Department, University of Calabria, via P. Bucci, Arcavacata di Rende, Italy

${ }^{20}$ European Southern Observatory, Karl-Schwarzschild-Str. 2, Garching, Germany

${ }^{21}$ Instituto de Astrofísica de Andalucía (IAA-CSIC), Granada, Spain

${ }^{22}$ Leiden Observatory, Leiden University, Leiden, The Netherlands

${ }^{23}$ GEPI-Observatoire de Paris Meudon. 5 Place Jules Jannsen, Meudon, France

${ }^{24}$ INAF/Osservatorio Astronomico di Brera, via Emilio Bianchi 46, Merate (LC), Italy

\begin{abstract}
Gamma-ray burst host galaxies are deficient in molecular gas, and show anomalous metal-poor regions close to GRB positions. Using recent Australia Telescope Compact Array (ATCA) Hi observations we show that they have substantial atomic gas reservoirs. This suggests that star formation in these galaxies may be fuelled by recent inflow of metal-poor atomic gas. While this process is debated, it can happen in low-metallicity gas near the onset of star formation because gas cooling (necessary for star formation) is faster than the HI-to- $\mathrm{H}_{2}$ conversion.
\end{abstract}

Keywords. galaxies: ISM, galaxies: formation, gamma rays: bursts, radio lines: galaxies 


\section{Introduction}

Galaxy formation models require significant gas inflow from the intergalactic medium to fuel star formation (Schaye et al. 2010). Indeed the current gas reservoirs in many galaxies are too low to sustain star formation (e.g. Draine 2009). Filamentary structures suggesting gas inflow have only been detected for two galaxies (Martin et al. 2014; Turner et al. 2015), so most of what we know about gas inflow is based on indirect evidence (Sancisi et al. 2008). In particular, metal-poor regions in inner parts of galaxies suggest recent accretion of metal-poor gas (Cresci et al. 2010; Sánchez Almeida et al. 2014).

Long gamma-ray bursts (GRBs) are explosions of very massive stars (e.g. Hjorth et al. 2003), so they pinpoint locations of recent star formation, which is usually believed to be fuelled by molecular gas $\left(\mathrm{H}_{2}\right.$; Carilli \& Walter 2013). However, GRB hosts were found to be deficient in molecular gas (Hatsukade et al. 2014; Stanway et al. 2015). Moreover, these galaxies often exhibit metal-poor regions close to the GRB positions (Christensen et al. 2008; Thöne et al. 2008, 2014; Levesque et al. 2011).

\section{HI survey of gamma-ray burst hosts: evidence for recent gas inflow}

These properties, together with large atomic gas (HI) masses reported in the first HI survey of GRB hosts (performed with ATCA; Michałowski et al. 2015) can be interpreted as a sign of recent metal-poor atomic gas inflow fuelling star formation giving rise to the GRB progenitor. Indeed, HI centroids are offsets towards the GRB locations, and in one case an optically dark Hi object is present $\sim 20 \mathrm{kpc}$ away from the GRB host, which can originate from inflowing gas. Moreover, the concentration of HI close to one GRB position was confirmed by follow-up observations of Arabsalmani et al. (2015).

Star formation fuelled by atomic gas can happen in recently-acquired metal-poor gas (even if the metallicity in other parts of a galaxy is higher), because gas cooling (necessary for star formation) is faster than the HI-to- $\mathrm{H}_{2}$ conversion (Krumholz 2012). GRB sites would then be expected to be metal-poor but relatively dusty (due to rapid dust production), consistent with observations (Hatsukade et al. 2014; Michałowski et al. 2014).

\section{References}

Arabsalmani, M., et al., 2015, MNRAS, 454, L51

Carilli, C. L., Walter, F., 2013, ARA\&A, 51, 105

Christensen, L., et al., 2008, A\&A, 490, 45

Cresci, G., et al., 2010, Nat, 467, 811

Draine, B. T., 2009, ASPC, 414, 453

Hatsukade, B., et al., 2014, Nat, 510, 247

Hjorth, J., et al., 2003, ApJ, 597, 699

Krumholz, M. R., 2012, ApJ, 759, 9

Levesque, E. M., et al., 2011, ApJ, 739, 23

Martin, D. C., et al., 2014, ApJ, 786, 106

Michałowski M. J., et al., 2014, A\&A, 562, A70

Michałowski M. J., et al., 2015, A\&A, 582, A78

Sánchez Almeida J., et al., 2014, ApJ, 783, 45

Sancisi, R., et al., 2008, A\&AR, 15, 189

Schaye, J., et al., 2010, MNRAS, 402, 1536

Stanway, E. R., et al., 2015, ApJ, 798, L7

Thöne C. C., et al., 2008, ApJ, 676, 1151

Thöne C. C., et al., 2014, MNRAS, 441, 2034

Turner, J. L., et al., 2015, Nat, 519, 331 